Société d'histoire de la révolution de 1848 et des

révolutions du XIXe siècle

49 | 2014

1814-1815. Expériences de la discontinuité

\title{
L'Archéologie linguistique du pouvoir et du peuple chez Augustin Thierry
}

A linguistic archeology of power and the people by Augustin Thierry

Linguistische Archäologie der Macht und des Volkes bei Augustin Thierry

\section{Aurélien Aramini}

\section{(2) OpenEdition}

Journals

Édition électronique

URL : http://journals.openedition.org/rh19/4760

DOI : $10.4000 /$ rh 19.4760

ISSN : $1777-5329$

Éditeur

La Société de 1848

Édition imprimée

Date de publication : 1 décembre 2014

Pagination : 179-193

ISSN : 1265-1354

Référence électronique

Aurélien Aramini, «L'Archéologie linguistique du pouvoir et du peuple chez Augustin Thierry », Revue d'histoire du XIXe siècle [En ligne], 49 | 2014, mis en ligne le 01 décembre 2014, consulté le 20 avril 2019. URL : http://journals.openedition.org/rh19/4760 ; DOI : 10.4000/rh19.4760 


\section{AURÉLIEN ARAMINI}

\section{L’Archéologie linguistique du pouvoir et du peuple chez Augustin Thierry}

Le peuple français ne dispose pas encore d'un récit historique dont il pourrait tirer «une leçon» susceptible de le guider «dans son rôle politique imminent ${ }^{1}$ : tel est le problème indissolublement épistémologique et politique qui s'impose à Augustin Thierry lorsqu'il collabore au Censeur Européen entre 1817 et 1820. L'historiographie de la France est alors dominée par l'ouvrage De la Monarchie française, "somme ultra-réactionnaire » que le Comte de Montlosier publie en 1814 et qui ramène "au centre du débat public la vieille thématique des invasions et des conquêtes $»^{3}$. Tandis que la Restauration se radicalise contre les Libéraux, Augustin Thierry inaugure la «révolution historique des années $1820 »^{4}$ dans une perspective scientifique et politique; bientôt, Guizot prononcera ses cours sur les origines du gouvernement représentatif et Barante commencera son Histoire des ducs de Bourgogne.

Publiées à partir du 13 juillet 1820 dans le Courrier Français, les Lettres sur l'histoire de France commencent un récit où la "généalogie nationale" se mue en "archéologie sociale»". Dans ces Lettres, Augustin Thierry fait de la "réforme des noms propres ${ }^{6}$ un aspect essentiel du renouveau de l'historiographie qu'il appelle de ses vœux. Loin d'être «une vaine prétention de style $»^{7}$, cette exigence linguistique participe d'un discours global dont les dimensions historique, linguistique, philosophique et politique s'articulent autour d'une pensée de l'origine et tout particulièrement de l'origine du pouvoir. Dans la huitième lettre de l'édition de 1827 des Lettres sur l'histoire de France, la dimension philologique du propos de l'historien s'enrichit en

1. Rulon Nephi Smithson, Augustin Thierry Social and Political Consciousness in the Evolution of a Historical Method, Genève, Droz, 1973, p. 59 (je traduis).

2. Marcel Gauchet, «Les Lettres sur l'histoire de France d'Augustin Thierry", in Pierre Nora [dir.], Les Lieux de mémoires, Paris, Gallimard, "Quarto», 1997, t. I, p. 805

3. Ibidem

4. Ibidem, p. 787.

5. Loïc Rignol, «Augustin Thierry et la politique de l'histoire. Genèse et principes d'un système de pensée", Revue d'histoire du XIX ${ }^{e}$ siècle, 25, 2002, p. 87-100, $\$ 11$.

6. Boris Réizov, L'Historiographie romantique française 1815-1830, Moscou, Éditions en Langues Étrangères, s. d., p. 162.

7. Ibidem, p. 161. 
reliant l'étude des langues à l'histoire du peuple ou plutôt des peuples. Au lieu de formuler in abstracto "l'acte par lequel un peuple est un peuple», Augustin Thierry envisage alors la possibilité de penser le peuple français en retraçant l'histoire de sa constitution à partir d'éléments hétérogènes identifiés par les langues sur un territoire précis à l'aube du Moyen Âge ${ }^{8}$.

Le sens de l'archéologie linguistique menée par Augustin Thierry apparaît tout particulièrement dans la réforme du nom de "Clovis» en "Hlodewig». Cette substitution patronymique est emblématique de la nature du lien qui se tisse durant la Restauration entre philologie, histoire et politique car elle possède une double dimension révélatrice du projet historiographique d'Augustin Thierry. La première dimension est épistémologique et concerne la vérité historique : la réforme du nom de "Clovis» en «Hlode-wig" permet de rendre aux temps mérovingiens «la vérité de couleur locale qui doit être le propre de l'histoire»'. La deuxième dimension est politique car la recherche de l'origine du pouvoir conduit à donner une consistance au peuple et à inscrire ses revendications révolutionnaires dans l'histoire de France en montrant que la Révolution est «tout à la fois rupture et commencement, mais aussi aboutissement prévisible de la longue marche du tiers état ${ }^{10}$. C'est dans cette perspective que l'historiographie d'Augustin Thierry appuyée sur l'étude des langues s'avère particulièrement féconde. S'insérant dans la séquence historique ouverte par «les événements révolutionnaires de 17891795, qui changent le principe de la souveraineté nationale et du même coup le principe du pouvoir linguistique ${ }^{11}$, l'archéologie du pouvoir et du peuple chez Augustin Thierry articule l'identité de la nation et la diversité linguistique. Dans cette perspective, la volonté "d'anéantir les patois" n'est donc pas nécessairement "une conséquence du système de nivellement et d'unité révolutionnaires ${ }^{12}$; elle traduirait plutôt un excès des députés révolutionnaires qui «frappèrent les existences locales, pour atteindre plus sûrement les pouvoirs injustes qu'elles soutenaient à côté des libertés légitimes » ${ }^{13}$.

8. Le lien entre l'histoire des langues et l'histoire des peuples vaincus n'apparaît pas dans les Lettres de 1820 qui se concentrent sur l'archéologie du pouvoir à travers l'analyse du titre de « roi». C'est dans le premier paragraphe de l'introduction de l'Histoire de la Conquête de l'Angleterre par les Normands (Paris, Firmin Didot, 1825, 3 vol., t. I, p. I et II) qu'Augustin Thierry formule explicitement pour la première fois l'hypothèse selon laquelle les dialectes et patois provinciaux sont les «traces vivantes» de la variété originelle des races sur le territoire national.

9. Augustin Thierry, Lettres sur l'histoire de France, lettre III, Paris, Sautelet, 1827, p. 21 (désormais cité LHF, suivi du numéro de la lettre et de la page). Les références aux lettres "originales» publiées dans le Courrier Français renvoient à l'édition d'Aude Déruelle (désormais citées LHF 1820 suivi du numéro de la lettre et de la page) : Augustin Thierry, Lettres sur l'histoire de France, édition critique par Aude Déruelle, Paris, Classiques Garnier, 2012.

10. François Hartog, "L'œil de l'historien et la voix de l'histoire», Communications, 43, 1986, p. 55-69, p. 56.

11. Renée Balibar, L'Institution du français Essai sur le colinguisme des Carolingiens à la République, Paris, PUF, coll. «Pratiques théoriques», 1985, p. 13.

12. Frédéric Auguste Ferdinand Thomas de Reiffenberg, «Nouvelles Observations sur les patois romans usités en Belgique", Bulletins de l'Académie Royale de Bruxelles, t. VI, n 8, p. 169-181, p. 170.

13. Augustin Thierry, Dix ans d'études historiques, Paris, Tessier, 1835, p. 266; ce texte est extrait d'un article publié dans le Censeur Européen le 2 février 1820. 
En effet, loin de conduire à "l'idéologie de Grégoire et de Barère» souhaitant "l'extinction "naturelle" du plurilinguisme populaire sous l'effet d'une propagation massive du français " ${ }^{14}$, les Lettres sur l'histoire de France contribuent à investir les langues régionales d'un sens révolutionnaire et à établir que l'histoire des langues, des dialectes et des patois provinciaux révèle l'identité plurielle du peuple dans les premières décennies du XIX siècle.

\section{L'ESSENCE DU POUVOIR DE L'ANCIEN RÉGIME RÉVÉLÉE PAR LES NOMS ET PAR LES MOTS}

Dès la parution de la première "Lettre sur l'histoire de France" publiée en 1820 dans Le Courrier Français, l'entreprise historiographique d'Augustin Thierry repose sur l'idée que l'histoire de la monarchie française, de Clovis jusqu'à la Révolution, prend sens relativement à une "prise de terre" où le dominium devient imperium. L'histoire politique de la France est d'abord territoriale. Pour cette raison, l'archéologie du pouvoir royal des Lettres sur l'histoire de France privilégie l'étude de la figure de «Clovis» - considéré comme le premier roi franc à régner en Gaule - au détriment de la figure de Pharamond auquel certains historiens, comme l'abbé Velly, confèrent pourtant le titre de premier roi de France ${ }^{15}$. L'historiographie d'Augustin Thierry pense le pouvoir de l'Ancien Régime à partir de la conquête d'un territoire où la lutte des classes ne fait que succéder à la lutte des races, les « relations réelles" configurant par conséquent les «relations personnelles " ${ }^{16}$. L'accaparement de la terre constitue le pouvoir et la dépossession des vaincus structure la société. Guerre raciale entre les Francs et les peuples gallo-romains au départ, ce phénomène est à l'origine de la relation sociale constitutive de l'Ancien Régime. Or, la «réforme des noms» donne à l'événement historique fondateur de l'histoire de France une signification particulière qui fait l'originalité de la théorie de la conquête dans l'historiographie d'Augustin Thierry ${ }^{17}$. Si Guizot

14. Renée Balibar, L'Institution du français, op. cit., p. 216.

15. Ainsi, dans son Histoire de France, depuis l'établissement de la monarchie jusquà Louis XIV (t. I, Paris, Saillant \& Nyon, 1770), l'abbé Velly considère que Pharamond, reconnu chef des Francs en 420, fonde la monarchie française parce qu' "on [lui] attribue communément [...] l'institution de la fameuse loi qui fut appelée Salique» (ibidem, p. 2). Cette conception «légale» du pouvoir ne retient pas l'attention d'Augustin Thierry qui privilégie la dimension «territoriale» de la royauté mérovingienne.

16. Je reprends la conceptualisation de Bruno Bernardi dans son article "Guerre, État, État de guerre : quand Schmitt lit Rousseau ", Philosophie, n 94, Paris, Les Éditions de Minuit, 2007, p. $52-65$.

17. L'historiographie "agonistique» d'Augustin Thierry a fait l'objet de nombreuses etudes : outre celles de Smithson, de M. Gauchet, de B. Réizov et de S. Mellon (The Political Uses of History, A Study of Historians in the French Restoration, Stanford University Press, Stanford, 1958), citons celles de Gheorgi Plékanov ("Augustin Thierry et la conception matérialiste de l'histoire», Le Devenir social, Paris, 1895), de Lionel Gossman («Augustin Thierry and Liberal historiography», History and Theory, Beiheft 15, Wesleyan University Press, Middletown, 1976, p. 3-83), de Keran Joseph Carroll (Some Aspects of the Historical Thought of Augustin Thierry (1795-1856), Washington, The Catholic University of America, 1951). 
commence, quelques mois après la publication de la première des Lettres sur l'histoire de France, un cours sur l'histoire des institutions ${ }^{18}$, le futur auteur de l'Histoire de la conquête de l'Angleterre trouve l'essence du pouvoir dans l'épaisseur des langues où se lit la différence des races. Sans être exclusives l'une de l'autre, les méthodes des deux grandes figures de l'historiographie romantique n'en ouvrent pas moins les deux voies théoriques que Michelet s'efforcera de faire converger une décennie plus $\operatorname{tard}^{19}$ : d'une part, une histoire politique où la civilisation se construit dans des formes d'organisation sociale et, d'autre part, une histoire des masses anonymes identifiées non seulement par le sang mais aussi par la langue.

Écrire la véritable histoire de la France suppose donc un «retour aux sources ${ }^{20}$. Mais les sources de l'histoire de France ne sont pas «françaises» ou, du moins, elles ne le sont pas encore. Pour faire le récit des temps mérovingiens, il ne suffit pas de parler français. Avec ironie, Augustin Thierry écrit qu’il aurait voulu conseiller à l'abbé Velly - qui «savait notre langue» mais qui "estropie tous les noms de notre histoire ${ }^{21}$ - "d'échanger toutes ces belles connaissances, contre l'intelligence d'une douzaine de mots germaniques ${ }^{22}$. En effet, l'histoire des temps mérovingiens doit être exhumée des textes où les déformations latines et françaises ont voilé l'origine et la nature du pouvoir qui se révèlent à travers l'étude des véritables noms utilisés au cours de l'histoire.

\section{L'étranger}

En distinguant dans le nom du "premier» roi de France les couches linguistiques qui se sont sédimentées au fil des siècles - du nom latinisé "Chlodovechus» des «chroniqueurs des premiers temps de la domination franke ${ }^{23}$ à celui de "Clovis» chez Nicole Gilles ${ }^{24}$ - Augustin Thierry identifie ce que la romanisation du nom a caché, à savoir l'invasion étrangère

18. Le cours d'histoire moderne que Guizot professe de 1820 à 1822 s'intitule «Histoire des origines du gouvernement représentatif et des institutions politiques de l'Europe depuis la chute de l'Empire romain jusqu'au XIV siècle».

19. Michelet revendiquera une méthode à «deux ailes» combinant "Nature et Esprit, deux interprétations du grand mouvement général» ("Préface de 1869", Histoire de France, nouvelle édition présentée par Paul Viallaneix et Paule Petitier, 17 volumes, Éditions des Équateurs, 2007, t. I, p. 14).

20. Cf. Aude Déruelle «introduction» aux Lettres sur l'histoire de France, op. cit., p. 45.

21. LHF, III, p. 25.

22. Ibidem (ibidem)

23. LHF, VII, p. 63. Les chroniques latines du IX ${ }^{\mathrm{e}}$ siècle, quant à elles, écrivent "Hludovicus"

24. Concernant la "francisation" du nom de Clovis, "les manuscrits des grandes chroniques de France, dites de Saint-Denis, marquent ce qu'on peut nommer le point de départ; on y voit pour les noms franks des variantes sans nombre dues à l'emploi arbitraire de la transcription d'après le latin et de la version en roman. On trouve, par exemple : Clodovées, Clodouvées et une fois seulement Cloovis [...]. Ce fut Nicole Gilles, auteur des Annales et Chroniques de France, l'historien populaire de la fin $\mathrm{XV}$ e siècle à celle du siècle suivant, qui, le premier, adopta sans variante le nom de Clovis " (Augustin Thierry, "Sur la restitution des noms propres dans la période germanique de l'histoire de France", lettre à Charles Nodier, en réponse à l'article publié dans la Revue de Paris «Diatribe du docteur Neophobus contre les fabricateurs de mots" (1842) in Augustin Thierry, Euvres complètes, t. VI, Paris, Furne, 1851, p. 365-389, p. 376-377). 
qui tient lieu d'origine de la société de l'Ancien Régime. "Hlode-wig ${ }^{25}$, tel fut le véritable nom du conquérant de la Gaule romaine. L'historien insiste d'abord sur le changement de tonalité produit par la romanisation d'un nom germanique : «le propre de la langue romane était d'altérer et d'adoucir les noms originairement tudesques ${ }^{26}$. Cependant, la transformation des noms réalisée par les chroniqueurs et les historiens de l'Ancien Régime n'est pas seulement une adaptation. Elle dissimule la violence de la conquête initiale qui vient rompre l'histoire de la Gaule : «Le nom de Clovis analogue à celui d'Amadis forme un véritable contresens avec les images rudes et sanglantes de la barbarie germaine ${ }^{27}$. Saluant la réforme des noms voulue par Augustin Thierry, Salvandy remarque justement qu'en écrivant "Gallawg", "Hlotwig», «Rik-hard» ou "Myrdhyn», "l'imagination retrouve les chefs ou les bardes farouches de hordes à demi-sauvages $(s i c)$, mieux que les noms amollis de Galgacus, de Clovis, de Richard, de Merlin l'enchanteur ${ }^{28}$.

Mais, pour l'essentiel, le propos d'Augustin Thierry n'insiste pas tant sur la violence de ces hordes que sur le fait qu'il s'agit de conquérants étrangers. "Hlode-wig", "Thiode-rik», "Hlode-her», "Mere-Wig»" ${ }^{29}$, ces noms de la "première race royale» sont avant tout des noms germaniques qui ne sonnent pas comme ceux des Gallo-Romains. La tonalité de leurs noms n'a rien de commun avec les patronymes policés par la civilisation qu'ont forgés "les premiers compilateurs français de notre histoire ${ }^{30}$ et que les historiens du XVIII siècle utilisent encore. Les véritables noms des rois mérovingiens où retentissent "des sons rudes et gutturaux d'un dialecte germanique " $^{31}$ révèlent que l'origine du pouvoir n'est autre que «l'asservissement et [...] la dépossession de tout un peuple par des étrangers ${ }^{32}$, étrangers de langue, de culture et de mœurs. À l'aube du Moyen Âge, le pouvoir qui donnera naissance à l'Ancien Régime ne constitue donc pas la France mais fracture celle-ci en imposant, de manière exogène, un partage progressif de la population de l'ancienne Gaule romaine entre un groupe allogène vainqueur et un groupe indigène $^{33}$ vaincu. Pour cette raison, la théorie de la conquête d'Augustin

25. Le nom «Hlode-wig» utilisé par Thierry est une reconstruction à partir du nom latin dont la racine est conservée («Chlodo» ou «Hlode») et le suffixe germanisé («vechus» donnant "wig»). Pour ce qui concerne le sens et l'utilisation du suffixe "wig», Augustin Thierry s'appuie sur la Deutsche Gammatik de Grimm ( $J$ Je me suis conformé pour cette explication, à l'opinion du savant Grimm, dans son excellente Grammaire de toutes les langues germaniques», LHF, seconde édition, Paris Sautelet et $\mathrm{C}^{\mathrm{ie}}, 1829$, p. 536).

26. LHF, XIV, p. 202.

27. Augustin Thierry, «Sur la restitution des noms propres dans la période germanique de l'histoire de France», op. cit., p. 389.

28. Salvandy cité par Boris Réizov, L'Historiographie romantique française 1815-1830, op. cit., p. 163.

29. LHF, V, p. 45.

30. LHF, II, p. 13.

31. LHF, I, p. 9.

32. LHF, IX, p. 85.

33. Le terme se trouve chez Augustin Thierry dans la neuvième lettre (LHF, IX, p. 85) ou encore dans la dixième lettre (LHF, X, p. 103). 
Thierry n'a pas pour catégories centrales le «fort» et le «faible», le "vainqueur" et le "vaincu", l'"aristocratie franque» et la "démocratie gauloise», même si ces catégories sont bien présentes : dans les Lettres sur l'histoire de France, les catégories centrales et originales sont plutôt celles de l'«étranger» et de l'«indigène» et c'est à partir d'elles que se déploie l'historiographie d'Augustin Thierry.

\section{Le roi nomade et barbare}

L'archéologie linguistique met en évidence non seulement l'origine étrangère du pouvoir mais aussi sa nature : si Clovis ne se nommait pas "Clovis» mais "Hlode-wig», il n'était pas non plus «roi» au sens du XVIII et du XIX ${ }^{e}$ siècle. Pour écrire l'histoire, la "contre-histoire " ${ }^{34}$ romantique commence par replacer les traces que livre le passé dans «les circonstances qui les ont fait naitre, et dont le tableau fidèle peut seul fixer le véritable sens ${ }^{35}$. Or il faut comprendre que "sous les vieux noms sont des choses neuves, et si la lettre demeure l'esprit change ${ }^{36}$. Ainsi en va-t-il du titre de "Roi». Dans la septième des Lettres sur l'histoire de France consacrée à l'examen philologique du titre de roi chez les Franks, Augustin Thierry montre que parler de «roi » à l'aube du Moyen Âge est un anachronisme conceptuel. Renvoyant à un pouvoir souverain monarchique qui concentre en une seule personne l'autorité politique, la signification moderne de «roi» diffère complètement de celle que les barbares germaniques accordaient au titre de «Koning». Traduit improprement par «rex», le titre de "Koning» correspondait à "l'idée vague de l'autorité, sans aucune détermination $»^{37}$ et pouvait désigner "un simple capitaine d'infanterie $»^{38}$. Du point de vue méthodologique, Augustin Thierry cherche ce sens archaïque aussi bien dans un chant médiéval germanique issu des "débris de la littérature des Franks de la Gaule » ${ }^{39}$ que dans l'analyse comparative des dialectes "teutoniques ${ }^{40}$ » - saxon, danois, suédois ou islandais - dialectes frères de celui des conquérants de la Gaule. Aussi l'historien trouve-t-il dans la culture linguistique la possibilité d'accéder aux significations de l'histoire en deçà des traces officielles. Mais la comparaison

34. Foucault, "Il faut défendre la société", cours au Collège de France (1975-1976), édition établie, dans le cadre de l'Association pour le centre Michel Foucault, sous la direction de François Ewald et Alessandro Fontana, par Mauro Bertani et Alessandro Fontana, coll. "Hautes Études», Seuil/Gallimard, 1997, p. 61. Même si l'étude développée par Foucault dans ce cours au Collège de France contribue davantage à l'élaboration d'une philosophie originale qu'à l'éclaircissement de l'historiographie romantique, elle n'en reste pas moins féconde pour mettre en évidence la modalité selon laquelle les concepts de la philosophie politique mis en œuvre au XVIII siècle - "pouvoir ", "peuple" et «langues» - vont être historicisés par les historiens romantiques (concernant la relation ambiguë entre Foucault et les historiens, $c f$. Arlette Farge, «Michel Foucault et les historiens : le malentendu", L'Histoire, $\mathrm{n}^{\circ} 154$, avril 1993, p. 74-76).

35. Augustin Thierry, Dix ans d'études historiques, op. cit., p. 157.

36. LHF, VI, p. 61.

37. LHF, VII, p. 68.

38. Ibidem, p. 68

39. Ibidem, p. 65.

40. Ibidem, p. 67. 
philologique ne peut devenir l'auxiliaire de l'histoire que parce que la conception des langues sous-jacente aux Lettres sur l'histoire de France participe d'un nouveau régime d'historicité dans lequel Schlegel, Bopp et Grimm ont fait entrer la philologie quelques années plus tôt : loin d'être des signes conventionnels, les mots sont, de part en part, traversés par l'histoire et portent en eux «l'esprit du peuple qui les a fait naître $»^{41}$. L'historien doit distinguer le sens différent des mots «Koning», «Rex» ou «Roi» parce qu'il devient évident qu'ils ne sont pas des représentations équivalentes d'une même réalité : ces termes expriment l'activité tout entière du peuple qui les utilise. En retrouvant «le vouloir fondamental $»^{42}$ des hordes germaniques qui anime la langue tudesque, Augustin Thierry comprend que Clovis n'a pas pu fonder la monarchie française et inaugurer l'histoire de France au sens où l'entendaient les historiens de l'Ancien Régime; "Clovis» était comme tous les «enfants de Mere-wig» un chef de nomades pillant "un pays civilisé» ${ }^{43}$. Et "Hlode-wig» n'incarne le pouvoir barbare que parce qu'il fait face à un peuple civilisé : comme l'explique Foucault, «il n'y a pas de barbare, s'il n'y a quelque part un point de civilisation par rapport auquel le barbare est extérieur, et contre lequel il vient se battre ${ }^{44}$. Ainsi, à partir de l'archéologie linguistique, le pouvoir de l'Ancien Régime se révèle-t-il être «étranger", "nomade» et "barbare ${ }^{45}$ ", en même temps que se constitue, en creux, le peuple «indigène» et " autochtone» qui, tout en étant absent des archives, a produit la civilisation. Le peuple ou plutôt «les peuples» comme le révèle l'histoire des langues vernaculaires qui foisonnent dans la France de la Restauration ${ }^{46}$.

\section{LA RECHERCHE DU PEUPLE DANS LES DIALECTES}

\section{ET LES PATOIS PROVINCIAUX}

De Boulainvilliers à Montlosier ${ }^{47}$, l'histoire des institutions a certes été étudiée à travers le prisme de la théorie de la conquête, mais Augustin Thierry mobilise celle-ci pour déplacer le centre de gravité du récit historique

41. Michel Foucault, Les mots et les choses, Paris, Gallimard, 1966, p. 231, p. 303.

42. Ibidem

43. LHF, XII, p. 126.

44. Michel Foucault, "Il faut défendre la société», op. cit., p. 174.

45. «Étranger et nomade» : comment ne pas voir dans cette conception du pouvoir mérovingien le miroir de la monarchie revenue de l'étranger et du retour des émigrés après la chute de l'Empire? En effet, «dans le contexte de la Restauration et de sa censure tatillonne, l'histoire médiévale fournit le prétexte inespéré à attaques prudentes mais claires contre le régime» (Philippe Martel, «Les historiens du début du XIX e siècle et le Moyen Âge occitan : Midi éclairé, Midi martyr ou Midi pittoresque", Romantisme, $1982, \mathrm{n}^{\circ} 35$, p. 49-72, p. 51).

46. Sur la situation linguistique de la France au XIX siècle où les langues foisonnent, $c f$. l'ouvrage d'Eugen Weber, La fin des terroirs, Paris, Fayard, 1983 [Peasants into Frenchmen, Stanford University Press, Stanford, 1976], tout particulièrement le sixième chapitre intitulé "Des langues à foison ".

47. Boris Réizov, L'Historiographie romantique française 1815-1830, op. cit., p. 130. Pour une étude des discours historiques et des dispositifs théoriques mobilisant la "lutte des races», $c f$. M. Foucault, "Il faut défendre la société", op. cit. 
vers les peuples conquis. Au XVIII siècle, dans l'histoire de l'abbé Velly ou du Père Daniel, «l'histoire des grands contient a fortiori l'histoire des petits »; l'historiographie de l'Ancien Régime repose sur le "postulat que l'histoire des forts emporte avec elle l'histoire des faibles ${ }^{48}$. L'histoire est essentiellement celle des grandes familles au pouvoir : pour le père Daniel, «l'Histoire d'un Royaume ou d'une Nation a pour objet le Prince ou l'État; c'est là comme le centre où tout doit tendre $\&$ se rapporter ${ }^{49}$. Avec la réforme des noms, les appellations barbares apparaissent immédiatement sur un fond de civilisation, celle de la Gaule romaine que les hordes germaniques viennent incendier. C'est l'histoire de cette civilisation incendiée et des peuples asservis qu'il faut écrire. L'historien de la France ne peut plus se contenter de la seule "histoire du peuple Frank " ${ }^{50}$ : l'histoire à écrire est celle des "dix peuples dont nous sommes fils " ${ }^{51}$ et que les "Sicambres massacrèrent, vendirent à l'encan ou attachèrent de corps et de biens, à la glèbe de leur conquête $»^{52}$. De la même manière que l'étude philologique a révélé l'origine et la nature du pouvoir de l'Ancien Régime, il doit être possible, selon Augustin Thierry, d'exhumer l'histoire du peuple français en étudiant la diversité des langues provinciales. C'est cette hypothèse féconde qu'expose la huitième des Lettres sur l'histoire de France dans l'édition de 1827. Tout en prolongeant la perspective philologique des Lettres publiées en $1820^{53}$, Augustin Thierry l'enrichit à partir des remarques développées dans l'introduction de l'Histoire de la Conquête de l'Angleterre par les Normands: la diversité des langues révèle non seulement la nature du pouvoir mais aussi l'origine - ou plutôt les origines du peuple français ${ }^{54}$.

\section{Les voix des vaincus}

Limmense majorité de la nation a été exclue des annales officielles. Où donc trouver ces "dix peuples dont nous sommes fils " 55 ? S'ils n'ont pas eu droit de cité, tous possédaient cependant une voix particulière dont l'écho nous parvient par-delà les siècles. Ce sont ces voix que les langues diverses de la France donnent à entendre. Elles représentent le témoignage permettant d'écrire notre histoire :

48. Michel Foucault, "Il faut défendre la société», op. cit., p. 61.

49. Gabriel Daniel, Histoire de France depuis l'établissement de la monarchie françoise dans les Gaules, tome 1, Paris, Denis Mariette, 1713, p. 33.

50. LHF, II, p. 12.

51. LHF, XI, p. 112.

52. LHF, II, 12.

53. Dans les Lettres originales, l'étude philologique de la notion de «roi» figure dans les cinquième et sixième lettres (LHF 1820, V et VI, p. 311 sqq.)

54. L'archéologie philologique du peuple à partir des patois et des dialectes ne figure pas explicitement dans les Lettres en 1820. Dans l'œuvre de Thierry, la première formulation du lien entre histoire des langues locales et histoire des peuples conquis apparaît dans l'Histoire de la Conquête de l'Angleterre par les Normands publié en 1825 .

55. LHF, XI, p. 112. 
Dès les temps les plus reculés, plusieurs populations de races différentes habitaient le territoire des Gaules : les Romains, quand ils envahirent ce pays, y trouvèrent trois peuples et trois langues. Quels étaient ces peuples, et quelle relation d'origine et de parenté se trouvaient-ils à l'égard des habitants des autres contrées de l'Europe? Y avait-il une race indigène, et dans quel ordre les autres races émigrées d'ailleurs étaient-elles venues se presser contre la première? Quel a été, dans la succession des temps, le mouvement de dégradation des différences primitives de mœurs, de caractère et de langage? En retrouve-t-on quelques vestiges dans les habitudes locales qui distinguent nos provinces, malgré la teinte d'uniformité répandue par la civilisation? Les dialectes et patois provinciaux, par les divers accidents de leurs vocabulaires et de leur prononciation, ne semblent-ils pas révéler une antique diversité d'idiomes? Enfin, cette inaptitude à prendre l'accent français, si opiniâtre chez nos compatriotes du midi, ne pourrait-elle pas servir à marquer la limite commune de deux races d'hommes anciennement distinctes? ${ }^{56}$

L'historien doit apprendre à entendre ces voix surgies du haut Moyen Âge. Dans l'épaisseur des langues provinciales est enfouie une histoire oubliée que le pouvoir n'a pas complètement réussi à occulter : la recherche des origines du peuple français dans les langues locales fait ressurgir des peuples qui ont été bâillonnés. Les langues vernaculaires sont les archives des dépossédés où s'est conservée l'histoire de ceux qui n'ont pas d'histoire. L'historien ne doit pas se contenter de voir, il doit aussi entendre : «si l'histoire est vision, elle est aussi audition : un mixte d'“œil" et d'"oreille" " "57. À l'écoute des sonorités locales, l'historien du peuple devra convertir «le dire des témoins en voir pour le lecteur ${ }^{58}$. Les langues provinciales et les patois seront ce «dire» qui fera "voir» la conquête originelle. Cependant, en s'enfonçant dans le particularisme des parlers locaux, l'historiographie des origines multiples de la France révélées par la diversité linguistique ne fissure-t-elle pas l'unité du peuple français et, partant, de l'histoire de France à laquelle les Lettres sont censées être une introduction?

L'enquête historique rencontre alors un problème politique qu'a dû affronter la Révolution française : la diversité des langues ne s'oppose-t-elle pas à l'unité de la nation? N'est-ce pas pour faire advenir politiquement le peuple, que la Révolution, à travers Grégoire et Barère, a voulu anéantir les patois? Et finalement, la situation babélienne de la France d'Ancien Régime n'est-elle pas l'une des causes de l'échec de la Révolution? C'est sur ce fond problématique qu'apparaissent l'originalité et la fécondité théorique de l'archéologie linguistique du pouvoir et du peuple dans l'historiographie libérale d'Augustin Thierry.

56. LHF, VIII, pp. 80-81.

57. François Hartog "L'œil de l'historien et la voix de l'histoire», art. cité, p. 55.

58. Ibidem 


\section{La Révolution, le peuple et les langues}

Entre 1789 et 1795, Révolution et monolinguisme ne s'impliquent pas mutuellement. Au contraire, "les tout premiers temps de la Révolution connaissent en effet une courte période de redécouverte du plurilinguisme en France. Nombre de textes politiques, notamment des lois et décrets, sont traduits du français en d'autres idiomes " $"$. Puis le rapport aux langues régionales va se lier progressivement à une certaine conception de la nation : plurilinguisme/fédéralisme ou monolinguisme/centralisme. Il est alors tentant de rattacher le discours d'Augustin Thierry aux «idées girondines désapprouvées si ardemment par les jacobins ${ }^{60}$ : la valorisation de la langue régionale, véhicule symbolique des traditions locales, s'inscrit globalement dans une conception fédéraliste. Dans la période qui s'ouvre avec la chute des Girondins, cette conception se heurte, au "discours jacobin" dont la radicalisation entraîne un "processus d'uniformisation et d'universalisation " ${ }^{61}$ s'étendant au domaine linguistique pour des raisons non seulement culturelles mais aussi politiques, liées à la poussée de la Contre-révolution. Cependant, l'opposition entre Augustin Thierry et Grégoire à propos du statut de la langue régionale n'est pas aussi tranchée qu'elle peut sembler l'être de prime abord. Pour l'un comme pour l'autre, la langue régionale a en effet une valeur historique et un statut épistémologique. Dans son rapport, Grégoire insiste sur l'intérêt d'une étude des dialectes car elle contribue à la connaissance de l'histoire des peuples et de l'esprit humain : «la connaissance des dialectes peut jeter du jour sur quelques monuments du moyen âge " ${ }^{62}$, permettre de remonter à «l'origine des nations» et éclairer l' « histoire des progrès de l'esprit humain " ${ }^{63}$. Toutefois, ce qui distingue l'abbé Grégoire de l'auteur des Lettres sur l'histoire de France, c'est le sens révolutionnaire actuel accordé à la langue régionale. Selon Grégoire, les dialectes sont les débris de la féodalité; l'attention qu'il porte aux langues régionales «sera surdéterminée par une histoire mythique du progrès, histoire centralisatrice dont le triomphe sur le patois aura pour signe de n'y plus entendre des voix mais d'y observer des pierres tombales ${ }^{64}$. À l'inverse, Augustin Thierry fait de la prononciation et du

59. Bärbel Plötner, «Langue littérature et identités nationales et régionales Jacob Grimm entre la France et l'Allemage Le cas breton ", in Michel Espagne et Michael Werner [dir.], Qu'est-ce qu'une littérature nationale? Approche pour une théorie interculturelle du champ littéraire, Paris, Éditions de la Maison des sciences de l'homme, 1994, p. 215.

60. Boris Réizov, L'Historiographie romantique française 1815-1830, op. cit., p. 156. Dans son étude sur Augustin Thierry, K. J. Carroll pense l'unité de la pensée libérale de la Restauration à partir de l'opposition "à la fois au Jacobinisme et à l'Ancien Régime» (Some Aspects..., tout particulièrement le deuxième chapitre, op. cit., p. 22, je traduis).

61. Bärbel Plötner, "Langue littérature et identités nationales et régionales Jacob Grimm entre la France et l'Allemage Le cas breton", op. cit., p. 216.

62. Grégoire, «Rapport sur la nécessité et les moyens d'anéantir les patois et d'universaliser l'usage de la langue française» dans Michel de Certeau, Dominique Julia et Jacques Revel, Une Politique de la langue, Paris, Éditions Gallimard, collection «folio histoire», 2002, p. 339.

63. Ibidem, p. 340.

64. Une Politique de la langue, op. cit., p. 79. 
vocabulaire "des dialectes et des patois provinciaux" les "traces vivantes ${ }^{65}$ de la perpétuité des races conquises et le signe de la présence même du peuple de la glèbe appelé à renverser le système issu de la conquête. Dans les Lettres sur l'histoire de France, les habitudes locales, dont les accents sont la couleur mélodique, doivent enrichir l'enquête de l'historien mais elles ne sont pas historicisées : terreau du patriotisme français, elles sont le lien vivant entre un présent pour lequel la Révolution est toujours d'actualité et un passé que l'historien doit reconstruire.

Cependant, cette diversité linguistique conçue comme la permanence d'une résistance au "génie de la conquête " ${ }^{66}$ ne conduit-elle pas Augustin Thierry à omettre le fait que la monarchie a pu favoriser l'expression provinciale? C'est en tout cas le discours de «la pensée réactionnaire, de Joseph de Maistre à Charles Maurras» qui a vu «dans l'ancienne monarchie la forme politique la mieux capable de respecter et de conforter les identités particulières des communautés organiques ${ }^{67}$. La pensée contre-révolutionnaire accorde indéniablement aux langues locales non seulement un intérêt historique mais aussi une valeur de vérité ${ }^{68}$. Cependant, il n'est pas certain que l'ancienne monarchie ait été respectueuse des identités particulières. En effet, Augustin Thierry montre que la politique monarchique opère déjà un important nivellement linguistique bien avant le rapport de Grégoire pour qui «l'unité de l'idiome est une partie intégrante de la révolution ${ }^{69}$. Par certains aspects, la politique de la langue de la Révolution française poursuit la politique de l'Ancien Régime. Durant les heures les plus sombres de la Révolution, la diversité des idiomes a contribué, sans nul doute, à affaiblir l'action de la Convention nationale ${ }^{70}$. Toutefois, loin d'être le fait de la Révolution, la destruction des langues provinciales imputée au jacobinisme par la pensée contre-révolutionnaire est engagée dès le Moyen Âge; loin d'avoir été favorisés par l'Ancien Régime, les idiomes locaux ne se sont maintenus que lorsque le pouvoir les laissait vivre ou ne les faisait pas mourir. Dans la troisième édition de l'Histoire de la conquête de l'Angleterre publiée en 1830, Augustin Thierry rappelle que la «langue d'oc» comme langue de culture périt au XIII siècle à la suite du rattachement contraint des provinces méri-

65. Histoire de la Conquête de l'Angleterre par les Normands, op. cit., p. I.

66. Augustin Thierry, Dix ans d'études historiques, op. cit., p. 297.

67. Jacques Revel, «La région» in Pierre Nora [dir.] Les Lieux de mémoire, 2, Éditions Gallimard coll. "Quarto", 1997, pp. 2907-2936, p. 2911.

68. Cf. Joseph de Maistre, tout particulièrement le "Deuxième entretien" des Soirées de SaintPétersbourg, Joseph de Maistre, Euvres, édition établie par Pierre Glaudes, Paris, Robert Laffont, 2007, p. 504.

69. Grégoire, «Rapport sur la nécessité et les moyens d'anéantir les patois et d'universaliser l'usage de la langue française» dans Une Politique de la langue, op. cit., p. 342.

70. Concernant le rapport entre la Révolution française et les langues régionales, $c f$. l'article de Brigitte Schlieben-Lange «La révolution française», première section du premier chapitre «Luttes sociales, dialectes, contraintes", in Sylvain Auroux [dir.] Histoire des idées linguistiques, t. III "L'hégémonie du comparatisme», Mardaga coll. «Philosophie et langage», 1999, p. 23-34. 
dionales à la France monarchique, dernier acte de la conquête "territoriale» qui s'étend du VIe au XIII siècle :

La vieille civilisation de ces provinces reçut un coup mortel par leur réunion forcée à des pays bien moins avancés en culture intellectuelle, en industrie et en politesse. C'est la plus désastreuse époque dans l'histoire des habitants de la France méridionale, que celle où ils devinrent Français, où le roi, que leurs aïeux avaient coutume d'appeler le roi de Paris, commença à les nommer eux-mêmes ses sujets de la langue d'oc, par opposition aux anciens Français d'outre-Loire, qui parlaient la langue d'oui. Depuis ce temps, la poésie classique du Midi, et même la langue qui lui était consacrée, périrent en Languedoc, en Poitou, en Limousin, en Auvergne et en Provence. À la place de cette langue des troubadours qui, sans contrainte politique, par le seul charme des ouvrages auxquels on la consacrait, s'était élevée dans tous ces pays au-dessus des idiomes locaux, il ne resta plus que des dialectes populaires, inélégants, incorrects surtout, ayant le défaut de n'être parfaitement compris que dans un rayon peu étendu ${ }^{71}$.

Les patois languedociens représentent les dernières survivances non du monde féodal mais d'une langue de culture étouffée par le pouvoir du «roi de Paris». Dans l'optique d'Augustin Thierry, les dialectes populaires du Languedoc ne sont pas les débris ou les témoins de la féodalité; ils sont les témoins vivants d'une ancienne résistance indigène au pouvoir des conquérants. Dans les replis de la langue, dans les accents régionaux est enfouie l'âme des peuples vaincus. La conquête les a dépossédés d'une identité dont les dialectes et les patois provinciaux conservent la trace : si l'histoire du pouvoir est territoriale, la "contre-histoire $»^{72}$ romantique du peuple est linguistique. Ce qui est en jeu dans l'appel à l'étude des contre-histoires locales dans l'ombre de l'histoire, c'est "la mémoire du groupe ${ }^{73}$ dont la langue particulière est dépositaire. Toutefois la volonté de donner consistance au peuple par l'étude des dialectes et des patois provinciaux ne détruit-elle pas l'idée même de peuple, "notion moniste par excellence ${ }^{74}$ ? La mémoire particulière du groupe n'entre-t-elle pas en contradiction avec l'unité de l'histoire de France?

\section{Histoire nationale contre histoire nationalitaire:} l'exemple de l'historiographie du Midi

Les Lettres sur l'histoire de France ont pu nourrir un type de discours contribuant à faire éclater l'idée même de "peuple français». Si les origines

71. Augustin Thierry, Histoire de la conquête de l'Angleterre, troisième édition revue et augmentée, quatre tomes, Paris, Mesnier, 1830, t. IV, p. 159.

72. Michel Foucault, "Il faut défendre la sociétée, op. cit., p. 57.

73. Christopher Lasch, Le seul et vrai paradis. Une histoire de l'idéologie du progrès et de ses critiques, traduit et présenté par Frédéric Joly, Champs Flammarion, 2006, p. 430.

74. Jean-Claude Caron, Frères de sang La guerre civile en France au XIXe siècle, Champ Vallon, coll. "La chose publique», 2009, p. 48. 
de la France donnent à voir des nations aux mœurs et aux langues différentes, qu'en est-il de la Nation française? Dès 1820, Augustin Thierry se voit reprocher par le Journal de Paris de vouloir "démembrer la France " 75 . Le cas de l'écriture de l'histoire du Midi permet cependant de préciser l'articulation entre diversité linguistique et identité nationale chez Augustin Thierry qui se revendique "Français et patriote ${ }^{76}$. Il est vrai que l'attention à l'histoire locale conduit l'historien à renforcer et justifier à l'aide "d'arguments anthropologiques la découverte [...] de la différence culturelle et nationale entre France du Nord et espace occitan ${ }^{77}$. De fait, le thème de la conquête élaboré par l'historien romantique sera mobilisé par des historiens comme Fauriel ${ }^{78}$ ou Mary-Lafon ${ }^{79}$ dont le travail a pu inspirer un «mouvement nationalitaire occitan ". Dans la perspective linguistique d'une construction du fait historique telle que la tracent les Lettres sur l'histoire de France, la maîtrise de la langue provinciale devient, chez les historiens occitans, la condition de la vérité historique : ainsi Charles de Portal fait-il de Mary-Lafon «un des rares écrivains qui aient bien compris l'histoire et la doctrine des Albigeois» en raison de "sa connaissance de la langue du peuple qu'il étudiait»" ${ }^{80}$. Cependant, le récit historique lui-même subit une complète transfiguration de l'historiographie romantique à l'historiographie «nationalitaire». Cette transfiguration apparait de manière particulièrement frappante dans les travaux de Gounon-Loubens. Dans la préface - en français - de l'ouvrage Les Vies des Troubadours écrites en roman par des auteurs du XIIIe siècle et traduites en français par un indigène ${ }^{81}$ publié à Magradoux en 1866, le Marquis de Gounon-Loubens, sous le pseudonyme de l'"Indigène", adopte un type de discours qui donne à voir le principe d'une historiographie "nationalitaire». Défendant l'identité nationale d'une "province» française à partir de l'affirmation de la multiplicité des origines nationales de la France, l'«Indigène» considère que le nom de "peuple français» devrait être entendu «dans le sens restreint qu'il faudrait toujours lui laisser ${ }^{82}$; la France est une «espèce de confédération, qui s'est organisée après la période d'oppression et de

75. LHF 1820, III, p. 304.

76. LHF 1820, VII, p. 323.

77. Philippe Martel, «Les historiens du début du XIXe siècle et le Moyen Âge occitan : Midi éclairé, Midi martyr ou Midi pittoresque», op. cit., p. 55.

78. Claude Charles Fauriel publie une Histoire de la Gaule méridionale sous la domination des conquérants germains, Paris, Paulin, 4 vol., 1836. En lieu et place d'une histoire de «France», Fauriel constitue la "Gaule méridionale» en objet historique et revendique le fait de s'être appuyé tout particulièrement sur une étude des langues, "point capital et décisif dans l'histoire de la civilisation et des mélanges des peuples" (Histoire de la Gaule méridionale, t. I, p. 544).

79. Les «revendications politiques" des "différents mouvements régionalistes méridionaux" «reposèrent tout entières sur le récit historique que Mary-Lafon avait mis en forme le premier, dès 1845, à partir des études romanes issues de Raynouard» (Sylvain Venayre, Les origines de la France, Quand les historiens racontaient la nation, Paris, Seuil, coll. «L'Univers historique», 2013, p. 168).

80. Philippe Martel, «Les historiens du début du XIX siècle et le Moyen Âge occitan : Midi éclairé, Midi martyr ou Midi pittoresque», op. cit., p. 49.

81. Marquis de Gounon-Loubens, Les Vies des Troubadours écrites en roman par des auteurs du XIII siècle et traduites en français par un indigène, Magradoux, Pierre D’En Sagnos, 1866.

82. Ibidem, p. XXVI. 
violence» et dont «le pouvoir est [...] toujours demeuré aux mains de la même nation, et précisément aux mains de celle qui avait le moins de caractère et d'intelligence politique ${ }^{83}$. Dans le discours de Gounon-Loubens, la multiplicité originelle des nations se substitue à l'unité d'un peuple français devenu introuvable. Certes, l'historiographie libérale et l'historiographie nationalitaire occitane mettent en œuvre une même théorie agonistique qui constitue «l'exact contrepied de celle que défendaient des idéologues ultras, justifiant par le droit de conquête les privilèges d'une aristocratie descendant des compagnons de Clovis ${ }^{84}$. Mais l'historiographie libérale et l'historiographie nationalitaire fonctionnent de deux manières radicalement différentes du point de vue du récit historique lui-même. L'histoire libérale «telle qu'on va la voir écrite par Guizot, Augustin Thierry, Thiers ${ }^{85}$, entrecroise deux grilles d'intelligibilité : la première consiste à «se donner, au départ, un rapport de force, un rapport de lutte ${ }^{86}$ et la deuxième fait du présent le moment de l'universel tenant lieu d' " achèvement totalisateur ${ }^{87}$ du parcours historique. Pour Augustin Thierry, faire appel à la mémoire linguistique du groupe ne fait pas obstacle à l'écriture d'une histoire nationale. La mémoire nationale s'enrichit d'une mémoire locale qui, en retour, prend sens dans l'histoire d'un peuple - qu'il soit du nord ou du sud de la Loire - en révolte contre ses conquérants. La diversité ethnique est immédiatement renvoyée à une structure agonistique qui la révèle : le Midi apparaît dans sa différence «raciale» dès lors qu'il devient peuple conquis - comme toutes les autres nations gallo-romaines - par un pouvoir étranger et nomade. L'historiographie nationalitaire, quant à elle, ne mobilise qu'une seule des deux grilles d'intelligibilité caractéristiques de l'historiographie libérale, à savoir celle du "commencement déchiré», sans l'articuler à celle de l'unité de la France tenant lieu de "vérité de l'universel ${ }^{88}$. Selon Augustin Thierry, l'identité française ne se construit pas contre l'origine linguistique particulière mais à partir d'elle : elle n'exclut pas la mémoire du groupe mais la réactive car «le premier mérite d'une histoire vraiment nationale écrite pour un grand peuple serait de n'oublier personne, de ne sacrifier personne, de présenter sur chaque portion du territoire les hommes et les faits qui lui appartiennent ${ }^{89}$.

La perspective ouverte par l'auteur des Lettres sur l'histoire de France laisse entrevoir la possibilité de renouveler la manière dont se pose le problème de l'articulation entre la diversité linguistique et l'unité de la nation. En prolongeant la réflexion d'Augustin Thierry, il serait légitime d'envisager que la

83. Ibidem, p.XXIV.

84. Ibidem, p. 56.

85. Michel Foucault, "Il faut défendre la sociétée, op. cit., p. 202.

86. Ibidem

87. Ibidem, p. 204.

88. Ibidem, p. 212. Pour Michel Foucault, c'est fondamentalement la question que l'histoire et la philosophie poseront conjointement au XIX ${ }^{\mathrm{e}}$ siècle : «qu'est-ce qui, dans le présent, porte l'universel?» (ibidem).

89. LHF, II, p. 12. 
véritable histoire de France doive reconnaitre le plurilinguisme passé et présent tout en étant téléologiquement orientée par la constitution d'un peuple où l'unité advient "à partir de singularités multiples ${ }^{90}:$ le monolinguisme jacobin conduisant à une situation de diglossie ${ }^{91}$ tout autant que l'affirmation nationalitaire ignorent le sens révolutionnaire des dialectes et des patois provinciaux ainsi que leur destination nationale. Dès lors, l'histoire de France ne peut être nationale que si elle est d'abord locale et elle ne sera nationale que lorsqu'elle aura rendu aux peuples vaincus la place qui leur revient - celle qui les constitue comme français - dans les annales "plurilingues» de la France.

Aurélien Aramini est docteur en philosophie de l'Université de Franche-Comté et chercheur associé au laboratoire Logiques de l'Agir, EA 2274.

90. Jacques Neefs, "Augustin Thierry : le moment de la "véritable" histoire de France», Romantisme, 1980, n $^{\circ} 28-29$, p. $289-303$, p. 291.

91. En cherchant à réaliser l'unité linguistique en anéantissant les dialectes, une telle politique de la langue produit une situation de «diglossie" où "une langue se voyant refuser tout espace officiel fonctionne dans une complète inexistence politique et institutionnelle», Sonia Branca-Rosoff et Nathalie Schneider, L'Écriture des citoyens Une analyse linguistique des peu-lettrés pendant la période révolutionnaire, Paris, Publications de l'INALF, coll. «Saint-Cloud», Klincksieck, 1994, p. 27. 